\title{
Observer-Based Stabilization of Spacecraft Rendezvous with Variable Sampling and Sensor Nonlinearity
}

\author{
Zhuoshi Li, ${ }^{1}$ Ming Liu, ${ }^{1}$ Hamid Reza Karimi, ${ }^{2}$ and Xibin Cao ${ }^{1}$ \\ ${ }^{1}$ Research Center of Satellite Technology, Harbin Institute of Technology, Harbin 150001, China \\ ${ }^{2}$ Department of Engineering, Faculty of Technology and Science, University of Agder, 4898 Grimstad, Norway \\ Correspondence should be addressed to Ming Liu; robustcontrol23@gmail.com
}

Received 10 July 2013; Accepted 19 August 2013

Academic Editor: Hongli Dong

Copyright (c) 2013 Zhuoshi Li et al. This is an open access article distributed under the Creative Commons Attribution License, which permits unrestricted use, distribution, and reproduction in any medium, provided the original work is properly cited.

\begin{abstract}
This paper addresses the observer-based control problem of spacecraft rendezvous with nonuniform sampling period. The relative dynamic model is based on the classical Clohessy-Wiltshire equation, and sensor nonlinearity and sampling are considered together in a unified framework. The purpose of this paper is to perform an observer-based controller synthesis by using sampled and saturated output measurements, such that the resulting closed-loop system is exponentially stable. A time-dependent Lyapunov functional is developed which depends on time and the upper bound of the sampling period and also does not grow along the input update times. The controller design problem is solved in terms of the linear matrix inequality method, and the obtained results are less conservative than using the traditional Lyapunov functionals. Finally, a numerical simulation example is built to show the validity of the developed sampled-data control strategy.
\end{abstract}

\section{Introduction}

Sampled-data systems, involving a continuous-time system and a discrete controller implemented via sample-and-hold elements, have received significant attention from control theorists mostly due to related performance problems which have arisen in practice [1-5]. Over the past few decades, three main approaches have been broadly employed for sampleddata systems [6-8]. The first one is the lifting technique method, wherein the investigated plant is converted into infinite-dimensional discrete system equivalently. The second one is the hybrid approach, where the sample-data plant is described in terms of hybrid discrete/continuous models. In this setting, the solution is derived via differential Riccati equations with jumps [9]. The third one is the input delay approach, wherein the digital control signal is represented as delayed input between two sampling instants [10, 11]. This approach has been extended to deal with nonuniform uncertain sampling $[12,13]$. Furthermore, this approach has been improved and incorporated with the impulsive system method to achieve less conservative results for stability analysis and controller/filter design [12]. Based on this approach, recently a new type of discontinuous Lyapunov functionals has been developed in $[14,15]$ to deal with sampled-data systems. In this approach, the values of the proposed discontinuous Lyapunov functionals are discontinuous at the input update times, but a decrease is guaranteed due to its special and interesting construction.

On the other hand, autonomous rendezvous becomes more and more significant for many future astronautic missions such as intercepting, repairing, saving, docking, large-scale structure assembling, and satellite networking. In the past decades considerable attention has been devoted to autonomous rendezvous, and a few results have been reported $[16,17]$. In fact, when the target orbit is approximately circular and the distance between them is much smaller than the orbit radius, the relative motion between two neighboring spacecrafts can be described by $\mathrm{C}-\mathrm{W}$ equations, which were introduced by Clohessey and Wiltshire $[16,18$, 19]. In recent years, based on the $\mathrm{C}-\mathrm{W}$ equations, a few results have been developed for the control problem of relative motion between two neighboring spacecraft [20,21]. In particular, the sampled-data control problem of relative position holding has been studied for spacecraft rendezvous with thrust nonlinearity [22]. 
In realistic engineering system, sensors and actuators cannot provide unlimited amplitude signal since the physical, safety or, technological constraints always occur and exhibit inevitably [23-26]. Due to the theoretical significance and practical importance of saturation phenomenon, the related problems of saturation filtering and control have been extensively investigated $[27,28]$. However, the associated results for sensor saturation have been relatively few which may be due to the technical difficulty [29-33]. On the other hand, in practical autonomous rendezvous, the simultaneous presence of sensor saturations and sampling is quite typical and exhibit frequently and is desired to be investigated for control and filtering problems. Although sampled-data control problem of autonomous rendezvous has begun to draw some research attention, there have been very limited papers that take sensor saturations and sampling phenomenon into account, and the purpose of this paper is therefore to shorten such a gap.

In this paper, we investigate the problem of observerbased guaranteed cost control of spacecraft rendezvous in the presence of nonuniform sampling and sensor saturation. Motivated by the aforementioned discontinuous Lyapunov functionals [12, 15], an improved time-dependent Lyapunov functional approach will be proposed in this paper. Sufficient conditions for the stability analysis of the closed-loop system and the controller design are provided in terms of the new designed Lyapunov functionals approach, which is further shown to have less conservativeness than the traditional approaches.

The remaining part of this paper is organized as follows. In Section 2, the problem of sampled-data control of spacecraft rendezvous is formulated. In Section 3, the sufficient condition for the exponential stability of the closed-loop system is provided, and the sufficient conditions for the existence of the sampled-data guaranteed cost controller are given. Finally, a simulation result is illustrated in Section 4 to show the validity of the developed control strategy.

\section{Problem Formulation}

In the orbital coordinate frame of chaser and target spacecrafts, it is well known that the origin attaches to the mass center of the target spacecraft; $x$-axis is along the vector from the earth center to the origin; $y$-axis is along the target orbit circumference; $z$-axis completes the right-handed frame; $r_{1}$ denotes the radius of the target circular orbit; $\mu$ denotes the gravitational parameter of the earth; $w$ denotes the angle velocity of the target; and $w=\left(\mu / r_{1}^{3}\right)^{1 / 2}$.

Based on Newton's motion theory, the relative dynamic model of chaser and target spacecrafts can be modeled by the following C-W equations:

$$
\begin{aligned}
\ddot{x}_{c}(t)= & 2 w \dot{y}_{c}(t)+w^{2}\left(r_{1}+x_{c}(t)\right) \\
& -\frac{\mu\left(r_{1}+x_{c}(t)\right)}{\left[\left(r_{1}+x_{c}(t)\right)^{2}+y_{c}^{2}(t)+z_{c}^{2}(t)\right]^{3 / 2}}+a_{x},
\end{aligned}
$$

$$
\begin{aligned}
\ddot{y}_{c}(t)= & -2 w \dot{x}_{c}(t)+w^{2} y_{c}(t) \\
& -\frac{\mu y_{c}(t)}{\left[\left(r_{1}+x_{c}(t)\right)^{2}+y_{c}^{2}(t)+z_{c}^{2}(t)\right]^{3 / 2}}+a_{y}, \\
\ddot{z}_{c}(t)=- & \frac{\mu y_{c}(t)}{\left[\left(r_{1}+x_{c}(t)\right)^{2}+y_{c}^{2}(t)+z_{c}^{2}(t)\right]^{3 / 2}}+a_{z},
\end{aligned}
$$

where $x_{c}(t), y_{c}(t)$, and $z_{c}(t)$ are the components of the relative position in corresponding axes and $a_{i}(i=x, y, z)$ is the $i$ th component of the control input vector. By the talar expansion and linearization approach, the linearized equation around the null solution is given by

$$
\begin{gathered}
\ddot{x}_{c}(t)-2 w \dot{y}_{c}(t)-3 w^{2} x_{c}(t)=\frac{1}{m} T_{x}, \\
\ddot{y}_{c}(t)+2 w \dot{x}_{c}(t)=\frac{1}{m} T_{y}, \\
\ddot{z}_{c}(t)+w^{2} z_{c}(t)=\frac{1}{m} T_{z},
\end{gathered}
$$

where $m$ is the mass of the chaser and $T_{i}(i=x, y, z)$ is the relative motion dynamic. We define the following vector and matrices:

$$
\begin{aligned}
& x(t)=\left[\begin{array}{llllll}
x_{c}^{T}(t) & y_{c}^{T}(t) & z_{c}^{T}(t) & \dot{x}_{c}^{T}(t) & \dot{y}_{c}^{T}(t) & \dot{z}_{c}^{T}(t)
\end{array}\right]^{T}, \\
& u(t)=\left[\begin{array}{lll}
T_{x}(t)^{T} & T_{y}(t)^{T} & T_{z}(t)^{T}
\end{array}\right]^{T}, \\
& y(t)=\left[\begin{array}{lll}
x_{c}(t)^{T} & y_{c}(t)^{T} & z_{c}(t)^{T}
\end{array}\right]^{T}, \\
& A=\left[\begin{array}{cccccc}
0 & 0 & 0 & 1 & 0 & 0 \\
0 & 0 & 0 & 0 & 1 & 0 \\
0 & 0 & 0 & 0 & 0 & 1 \\
3 w^{2} & 0 & 0 & 0 & 2 w & 0 \\
0 & 0 & 0 & -2 w & 0 & 0 \\
0 & 0 & -w^{2} & 0 & 0 & 0
\end{array}\right], \\
& B=\frac{1}{m}\left[\begin{array}{lll}
0 & 0 & 0 \\
0 & 0 & 0 \\
0 & 0 & 0 \\
1 & 0 & 0 \\
0 & 1 & 0 \\
0 & 0 & 1
\end{array}\right]
\end{aligned}
$$

then the C-W equation (1) can be described equivalently as follows:

$$
\begin{gathered}
\dot{x}(t)=A x(t)+B u(t), \\
y(t)=C x(t) .
\end{gathered}
$$

As seen in (5), the specific relative motion of the chaser and target can be prevailed by designing $u(t)$.

It is supposed that the signals are sampled before transmitted to the controller side. Define $\mathscr{T} \triangleq\left\{t_{1}, t_{2}, t_{3}, \ldots\right\}$ as a strictly increasing sequence of sampling times in $\left(t_{0}, \infty\right)$ for 
initial time $t_{0}=0$. We also assume that the sampling internals between any two neighbour sampling instants satisfy

$$
t_{k+1}-t_{k} \leq h, \quad \forall k>0,
$$

where $h>0$ is a known constant. Therefore, the signal received in the observer and controller side is of the form $y\left(t_{k}\right)$ at the sampling instant $t_{k}$. The observer dynamics are thus designed as follows:

$$
\dot{\hat{x}}(t)=A \hat{x}(t)+B u(t)+L\left(y\left(t_{k}\right)-C \hat{x}(t)\right) .
$$

Define the error vector as

$$
e(t)=\widehat{x}-x(t)
$$

then we have

$$
\dot{e}(t)=(A-L C) e(t)+L C\left(x\left(t_{k}\right)-x(t)\right) .
$$

During the orbital transfer process, the thrust constraint always satisfies the following:

$$
\left|u_{i}(t)\right| \leqslant u_{i, \max } \quad(i=x, y, z),
$$

where $u_{i}(t)$ is the control input thrust along the $i$ th axis at the sampling instant $k$ and $u_{i \text {, max }}$ is the maximum allowed thrust along the $i$ th axis.

As pointed out in Section 1, a large number of realistic engineering applications will inevitably result in the nonlinear characteristic of sensors. Therefore, in this work, we use $\phi(\cdot): \mathbb{R}^{m} \rightarrow \mathbb{R}^{m}$ to denote the actuator nonlinearity, which belongs to $\left[F_{1}, F_{2}\right]$ for some given diagonal matrices $F_{1} \in \mathbb{R}^{m \times m}, F_{2} \in \mathbb{R}^{m \times m}$ with $F_{1} \geq 0, F_{2} \geq 0$ and $F_{2}>F_{1}$. In particular, $\phi(\cdot)$ satisfies the following sector condition:

$$
\begin{array}{r}
\left(\phi(u(t))-F_{1} u(t)\right)^{T}\left(\phi(u(t))-F_{2} u(t)\right) \leq 0, \\
\forall u(t) \in \mathbb{R}^{m} .
\end{array}
$$

In light of (11), the nonlinear function $\phi(u(t))$ can be decomposed into a linear and a nonlinear part:

$$
\phi(u(t))=F_{1} u(t)+\phi_{s}(u(t)),
$$

and the nonlinearity $\phi_{s}(u(t))$ satisfies $\phi_{s}(u(t)) \in \Phi_{s}$, where the set $\Phi_{s}$ is defined as

$$
\begin{array}{r}
\Phi_{s} \triangleq\left\{\phi_{s}: \phi_{s}^{T}(u(t))\left(\phi_{s}(u(t))-F u(t)\right) \leq 0\right\}, \\
F \triangleq F_{2}-F_{1} .
\end{array}
$$

Substituting (12) into (5) yields

$$
\dot{x}(t)=A x(t)+B F_{1} u(t)+B \phi_{s}(u(t)) .
$$

Designing the control scheme as

$$
u(t)=K \hat{x}(t)
$$

one can obtain

$$
\dot{x}(t)=\left(A+B F_{1} K\right) x(t)+B F_{1} K e(t)+B \phi_{s}(u(t)) .
$$

As a result, the following overall closed-loop system is obtained:

$$
\begin{aligned}
& \dot{x}(t)=\left(A+B F_{1} K\right) x(t)+B F_{1} K e(t)+B \phi_{s}(u(t)), \\
& \dot{e}(t)=(A-L C) e(t)+L C\left(x\left(t_{k}\right)-x(t)\right) .
\end{aligned}
$$

Consider the following performance index function:

$$
J(t)=\int_{0}^{\infty}\left[x^{T}(t) U x(t)+u^{T}(t) W u(t)\right] d t
$$

where $U>0$ and $W>0$ are known matrices with appropriate dimensions.

The objective of this paper now can be formulated as follows.

Problem 1. Design a sampled-data control law $u\left(t_{k}\right)$, such that the following conditions are met:

(1) the closed-loop system (17) is exponentially stable with a given decay rate $\alpha>0$;

(2) the control input satisfies the given upper bound (10);

(3) the performance index function (18) has an upper bound.

\section{Main Results}

In this Section, we will first perform the stability analysis and control scheme design for system (17). Furthermore, we will present the design scheme of the guaranteed cost controller for system (17). Consider the system (17), we design the state feedback gain $K$ and observer gain $L$ such that $A+B K$ and $A-L C$ are Hurwitz. We provide the following Theorem.

Theorem 2. Consider the closed-loop system (17) with $K$ and $L$ designed previously, given a decay rate $\alpha>0$; if there exist positive definite matrices $Q_{1}, Q_{2}, Q_{3}$, and $P_{1} \in \mathbb{R}^{9 \times 9}$, and matrices $S_{1}, S_{2}, T_{1}, T_{2}$, and $T_{3} \in \mathbb{R}^{9 \times 9}$ such that the following matrices conditions hold:

$$
\begin{aligned}
& \Pi=\left[\begin{array}{cccccc}
\Pi_{11} & \Pi_{12} & \Pi_{13} & \Pi_{14} & 0 & \Pi_{16} \\
\star & \Pi_{22} & \Pi_{23} & \Pi_{24} & 0 & \Pi_{26} \\
\star & \star & \Pi_{33} & \Pi_{34} & 0 & 0 \\
\star & \star & \star & \Pi_{44} & \Pi_{45} & \Pi_{46} \\
\star & \star & \star & \star & \Pi_{55} & 0 \\
\star & \star & \star & \star & \star & -I_{m}
\end{array}\right]<0, \\
& \widehat{\Pi}=\left[\begin{array}{ccccccc}
\widehat{\Pi}_{11} & \widehat{\Pi}_{12} & \widehat{\Pi}_{13} & \widehat{\Pi}_{14} & \widehat{\Pi}_{15} & 0 & \widehat{\Pi}_{17} \\
\star & \widehat{\Pi}_{22} & \widehat{\Pi}_{23} & \widehat{\Pi}_{24} & \widehat{\Pi}_{25} & 0 & \widehat{\Pi}_{27} \\
\star & \star & \widehat{\Pi}_{33} & \widehat{\Pi}_{34} & \widehat{\Pi}_{35} & 0 & 0 \\
\star & \star & \star & \widehat{\Pi}_{44} & 0 & 0 & 0 \\
\star & \star & \star & \star & \widehat{\Pi}_{55} & \widehat{\Pi}_{56} & \widehat{\Pi}_{57} \\
\star & \star & \star & \star & \star & \widehat{\Pi}_{66} & 0 \\
\star & \star & \star & \star & \star & \star & -I_{m}
\end{array}\right]<0, \\
& {\left[\begin{array}{cc}
u_{i, \max }^{-2} K^{T} R_{i}^{T} R_{i} K & u_{i, \max }^{-2} K^{T} R_{i}^{T} R_{i} K \\
\star & u_{i, \max }^{-2} K^{T} R_{i}^{T} R_{i} K
\end{array}\right]} \\
& -\left[\begin{array}{cc}
\rho^{-1} Q_{1} & 0 \\
\star & \rho^{-1} P_{1}
\end{array}\right]<0,
\end{aligned}
$$


where

$$
\begin{aligned}
& \Pi_{11}=2 \alpha Q_{1}+2 \alpha h Q_{3}-T_{1}-T_{1}^{T}-M_{1}^{T}\left(A+B F_{1} K\right) \\
& -\left(A+B F_{1} K\right)^{T} M_{1} \text {, } \\
& \Pi_{12}=Q_{1}+h Q_{3}+M_{1}^{T} \text {, } \\
& \Pi_{13}=Q_{3}-2 \alpha h Q_{3}+T_{1}^{T} \text {, } \\
& \Pi_{14}=S_{1}-M_{1}^{T} B F_{1} K \text {, } \\
& \Pi_{16}=K^{T} F^{T}-M_{1}^{T} B, \\
& \Pi_{22}=h Q_{2}+M_{2}+M_{2}^{T}, \\
& \Pi_{23}=-h Q_{3}+T_{2}^{T} \text {, } \\
& \Pi_{24}=-M_{2}^{T} B F_{1} K \text {, } \\
& \Pi_{26}=-0.5 M_{2}^{T} B \text {, } \\
& \Pi_{33}=-Q_{3}+2 \alpha h Q_{3}+T_{3}+T_{3}^{T}, \\
& \Pi_{34}=-C^{T} L^{T} S_{1} \text {, } \\
& \Pi_{44}=2 \alpha P_{1}-S_{1}^{T}(A-L C)-(A-L C)^{T} S_{1}, \\
& \Pi_{45}=P_{1}+S_{1}^{T}, \\
& \Pi_{46}=0.5 K^{T} F^{T} \text {, } \\
& \Pi_{55}=S_{2}+S_{2}^{T}, \\
& \widehat{\Pi}_{11}=2 \alpha Q_{1}-T_{1}-T_{1}^{T}-M_{1}^{T}\left(A+B F_{1} K\right) \\
& -\left(A+B F_{1} K\right)^{T} M_{1} \text {, } \\
& \widehat{\Pi}_{12}=Q_{1}+M_{1}^{T} \text {, } \\
& \widehat{\Pi}_{13}=Q_{3}+T_{1}^{T} \text {, } \\
& \widehat{\Pi}_{14}=h T_{1}^{T} \text {, } \\
& \widehat{\Pi}_{15}=S_{1}-M_{1}^{T} B F_{1} K \text {, } \\
& \widehat{\Pi}_{17}=K^{T} F^{T}-M_{1}^{T} B_{1} \text {, } \\
& \widehat{\Pi}_{22}=M_{2}+M_{2}^{T} \text {, } \\
& \widehat{\Pi}_{23}=T_{2}^{T} \text {, } \\
& \widehat{\Pi}_{24}=h T_{2}^{T} \text {, } \\
& \widehat{\Pi}_{25}=-M_{2}^{T} B F_{1} K \text {, } \\
& \widehat{\Pi}_{27}=-0.5 M_{2}^{T} B \text {, } \\
& \widehat{\Pi}_{33}=-Q_{3}+T_{3}+T_{3}^{T} \text {, } \\
& \widehat{\Pi}_{34}=h T_{3}^{T} \text {, }
\end{aligned}
$$

$\widehat{\Pi}_{35}=-C^{T} L^{T} S_{1}$,

$\widehat{\Pi}_{44}=-e^{-2 \alpha h} h Q_{2}$,$$
\widehat{\Pi}_{55}=2 \alpha P_{1}-S_{1}^{T}(A-L C)-(A-L C)^{T} S_{1}^{T} \text {, }
$$$$
\widehat{\Pi}_{56}=P_{1}+S_{1}^{T} \text {, }
$$$$
\widehat{\Pi}_{57}=0.5 K^{T} F^{T} \text {, }
$$$$
\widehat{\Pi}_{66}=S_{2}+S_{2}^{T} \text {, }
$$

then system (17) is exponentially stable with the decay rate $\alpha>$ 0 .

Proof. For system (17), consider the following Lyapunov functional: $V(t)=V_{1}(t)+V_{2}(t)+V_{3}(t)+V_{4}(t)$, where

$$
\begin{aligned}
& V_{1}(t)=x^{T}(t) Q_{1} x(t), \\
& V_{2}(t)=\left(h-t+t_{k}\right) \int_{t_{k}}^{t} \dot{x}^{T}(s) Q_{2} \dot{x}(s) e^{-2 \alpha(t-s)} d s, \\
& V_{3}(t)=\left(h-t+t_{k}\right)\left(x\left(t_{k}\right)-x(t)\right)^{T} Q_{3}\left(x\left(t_{k}\right)-x(t)\right), \\
& V_{4}(t)=e^{T}(t) P_{1} e(t) .
\end{aligned}
$$

In formula (4), $Q_{i}>0(i=1,2,3)$ and $P_{1}>0$ are Lyapunov matrices to be designed, and $\alpha>0$ is the decay date as previous defined. It can be calculated that

$$
\begin{aligned}
V_{1}(t)+ & 2 \alpha V_{1}(t)=2 \dot{x}^{T}(t) Q_{1} x(t)+2 \alpha x^{T}(t) Q_{1} x(t), \\
\dot{V}_{2}(t)= & -e^{-2 \alpha t} \int_{t_{k}}^{t} \dot{x}^{T}(s) Q_{2} \dot{x}(s) e^{2 \alpha s} d s \\
& +\left(h-t+t_{k}\right) e^{-2 \alpha t}(-2 \alpha) \int_{t_{k}}^{t} \dot{x}^{T}(s) Q_{2} \dot{x}(s) e^{2 \alpha s} d s \\
= & \left(h-t+t_{k}\right) e^{-2 \alpha t} \\
& \times\left(\dot{x}^{T}(t) Q_{2} \dot{x}(t) e^{2 \alpha t}-\dot{x}^{T}\left(t_{k}\right) Q_{2} \dot{x}\left(t_{k}\right) e^{2 \alpha t_{k}}\right) .
\end{aligned}
$$

Notice that $\dot{x}^{T}\left(t_{k}\right) Q_{2} \dot{x}\left(t_{k}\right) e^{2 \alpha t_{k}}=0$; thus

$$
\begin{aligned}
\dot{V}_{2}(t)= & -e^{-2 \alpha t} \int_{t_{k}}^{t} \dot{x}^{T}(s) Q_{2} \dot{x}(s) e^{2 \alpha s} d s \\
& -2 \alpha\left(h-t+t_{k}\right) e^{-2 \alpha t} \int_{t_{k}}^{t} \dot{x}^{T}(s) Q_{2} \dot{x}(s) e^{2 \alpha s} d s \\
& +\left(h-t+t_{k}\right) \dot{x}^{T}(t) Q_{2} \dot{x}(t) .
\end{aligned}
$$


Therefore it is derived that

$$
\begin{aligned}
\dot{V}_{2}(t)+ & 2 \alpha V_{2}(t) \\
= & -e^{-2 \alpha t} \int_{t_{k}}^{t} \dot{x}^{T}(s) Q_{2} \dot{x}(s) e^{2 \alpha s} d s \\
& -2 \alpha\left(h-t+t_{k}\right) e^{-2 \alpha t} \int_{t_{k}}^{t} \dot{x}^{T}(s) Q_{2} \dot{x}(s) e^{2 \alpha s} d s \\
& +\left(h-t+t_{k}\right) \dot{x}^{T}(t) Q_{2} \dot{x}(t) \\
& +2 \alpha\left(h-t+t_{k}\right) e^{-2 \alpha t} \int_{t_{k}}^{t} \dot{x}^{T}(s) Q_{2} \dot{x}(s) e^{2 \alpha s} d s \\
= & -e^{-2 \alpha t} \int_{t_{k}}^{t} \dot{x}^{T}(s) Q_{2} \dot{x}(s) e^{2 \alpha s} d s \\
& +\left(h-t+t_{k}\right) \dot{x}^{T}(t) Q_{2} \dot{x}(t) .
\end{aligned}
$$

Considering $V_{3}(t)$, it is shown that

$$
\begin{aligned}
\dot{V}_{3}(t)= & -\left(x\left(t_{k}\right)-x(t)\right)^{T} Q_{3}\left(x\left(t_{k}\right)-x(t)\right) \\
& -\left(h-t+t_{k}\right) \dot{x}^{T}(t) Q_{3}\left(x\left(t_{k}\right)-x(t)\right) \\
& -\left(h-t+t_{k}\right)\left(x\left(t_{k}\right)-x(t)\right)^{T} Q_{3} \dot{x}(t),
\end{aligned}
$$

$$
\begin{aligned}
\dot{V}_{3}(t) & +2 \alpha V_{3}(t) \\
= & -\left(x\left(t_{k}\right)-x(t)\right)^{T} Q_{3}\left(x\left(t_{k}\right)-x(t)\right) \\
& -\left(h-t+t_{k}\right) \dot{x}^{T}(t) Q_{3}\left(x\left(t_{k}\right)-x(t)\right) \\
& -\left(h-t+t_{k}\right)\left(x\left(t_{k}\right)-x(t)\right)^{T} Q_{3} \dot{x}(t) \\
& +2 \alpha\left(h-t+t_{k}\right)\left(x\left(t_{k}\right)-x(t)\right)^{T} Q_{3}\left(x\left(t_{k}\right)-x(t)\right) .
\end{aligned}
$$

For $V_{4}(t)$, it can be calculated that

$$
V_{4}(t)+2 \alpha V_{4}(t)=2 \dot{e}^{T}(t) P_{1} e(t)+2 \alpha e^{T}(t) P_{1} e(t) .
$$

As a result, we have

$$
\begin{aligned}
\dot{V}(t) & +2 \alpha V(t) \\
= & \dot{V}_{1}(t)+2 \alpha V_{1}(t)+\dot{V}_{2}(t)+2 \alpha V_{2}(t) \\
& +\dot{V}_{3}(t)+2 \alpha V_{3}(t)+\dot{V}_{4}(t)+2 \alpha V_{4}(t) \\
\leqslant & 2 \dot{x}^{T}(t) Q_{1} x(t)+2 \alpha x^{T}(t) Q_{1} x(t) \\
& -e^{-2 \alpha t} \int_{t_{k}}^{t} \dot{x}^{T}(s) Q_{2} \dot{x}(s) e^{2 \alpha s} d s \\
& +\left(h-t+t_{k}\right) \dot{x}^{T}(t) Q_{2} \dot{x}(t) \\
& -\left(x\left(t_{k}\right)-x(t)\right)^{T} Q_{3}\left(x\left(t_{k}\right)-x(t)\right)
\end{aligned}
$$

$$
\begin{aligned}
& -\left(h-t+t_{k}\right) \dot{x}^{T}(t) Q_{3}\left(x\left(t_{k}\right)-x(t)\right) \\
& -\left(h-t+t_{k}\right)\left(x\left(t_{k}\right)-x(t)\right)^{T} Q_{3} \dot{x}(t) \\
& +2 \alpha\left(h-t+t_{k}\right)\left(x\left(t_{k}\right)-x(t)\right)^{T} Q_{3}\left(x\left(t_{k}\right)-x(t)\right) \\
& +2 \dot{e}^{T}(t) P_{1} e(t)+2 \alpha e^{T}(t) P_{1} e(t) .
\end{aligned}
$$

We define the following variable:

$$
\eta_{x}(t)=\frac{1}{t-t_{k}} \int_{t_{k}}^{t} \dot{x}(s) d s,
$$

and it is derived that

$$
\begin{aligned}
e^{-2 \alpha t} \int_{t_{k}}^{t} e^{2 \alpha s} \dot{x}^{T}(s) Q_{2} \dot{x}(s) d s \\
\geqslant e^{-2 \alpha t} e^{2 \alpha t_{k}} \int_{t_{k}}^{t} \dot{x}^{T}(s) Q_{2} \dot{x}(s) \\
=e^{-2 \alpha\left(t-t_{k}\right)} \int_{t_{k}}^{t} \dot{x}^{T}(s) Q_{2} \dot{x}(s) .
\end{aligned}
$$

By Jensen's inequality, it can be shown that

$$
\begin{aligned}
& \int_{t_{k}}^{t} \dot{x}^{T}(s) Q_{2} \dot{x}(s) d s \\
& \leqslant \frac{1}{t-t_{k}} \int_{t_{k}}^{t} \dot{x}^{T}(s) d s Q_{2} \int_{t_{k}}^{t} \dot{x}(s) d s \\
& =\left(t-t_{k}\right)\left(\frac{1}{t-t_{k}} \int_{t_{k}}^{t} \dot{x}^{T}(s) d s\right) \\
& \quad \times Q_{2}\left(\frac{1}{t-t_{k}} \int_{t_{k}}^{t} \dot{x}(s) d s\right) \\
& =\left(t-t_{k}\right) \eta_{x}^{T}(t) Q_{2} \eta_{x}(t) .
\end{aligned}
$$

In particular, for $t-t_{k}=0$, it can be verified that the following holds:

$$
\begin{aligned}
& \left.\frac{1}{t-t_{k}} \int_{t-t_{k}}^{t} \dot{x}(s) d s\right|_{t-t_{k}=0} \\
& \quad=\lim _{\left(t-t_{k}\right) \rightarrow 0} \frac{1}{t-t_{k}} \int_{t_{k}}^{t} \dot{x}(s) d s=\dot{x}(t) .
\end{aligned}
$$

Therefore, it is shown from (30) that

$$
\begin{aligned}
\dot{V}(t)+ & 2 \alpha V(t) \\
\leqslant & 2 \dot{x}^{T}(t) Q_{1} x(t)+2 \alpha x^{T}(t) Q_{1} x(t) \\
& -e^{-2 \alpha\left(t-t_{k}\right)} \tau(t) \eta_{x}^{T}(t) Q_{2} \eta_{x}(t) \\
& +\left(h-t+t_{k}\right) \dot{x}^{T}(t) Q_{2} \dot{x}(t)
\end{aligned}
$$




$$
\begin{aligned}
& -\left(x\left(t_{k}\right)-x(t)\right)^{T} Q_{3}\left(x\left(t_{k}\right)-x(t)\right) \\
& -\left(h-t+t_{k}\right) \dot{x}^{T}(t) Q_{3}\left(x\left(t_{k}\right)-x(t)\right) \\
& -\left(h-t+t_{k}\right)\left(x\left(t_{k}\right)-x(t)\right)^{T} Q_{3} \dot{x}(t) \\
& +2 \alpha\left(h-t+t_{k}\right)\left(x\left(t_{k}\right)-x(t)\right)^{T} Q_{3}\left(x\left(t_{k}\right)-x(t)\right) \\
& +2 \dot{e}^{T}(t) P_{1} e(t)+2 \alpha e^{T}(t) P_{1} e(t) .
\end{aligned}
$$

We now insert free-weighting matrices by introducing the following zero terms:

$$
\begin{aligned}
& 2\left[e^{T}(t) S_{1}^{T}+\dot{e}^{T}(t) S_{2}^{T}\right] \\
& \quad \times\left[\dot{e}(t)-(A-L C) e(t)-L C\left(x\left(t_{k}\right)-x(t)\right)\right]=0, \\
& 2\left[x^{T}(t) T_{1}^{T}+\dot{x}^{T}(t) T_{2}^{T}+x^{T}\left(t_{k}\right) T_{3}^{T}\right] \\
& \quad \times\left[-x(t)+x\left(t_{k}\right)+\left(t-t_{k}\right) \eta_{x}(t)\right]=0, \\
& 2\left[x^{T}(t) M_{1}^{T}+\dot{x}^{T}(t) M_{2}^{T}\right] \\
& \quad \times\left[\dot{x}(t)-\left(A+B F_{1} K\right) x(t)-B F_{1} K e(t)-B \phi_{s}(u(t))\right]=0,
\end{aligned}
$$

where the free-weighting matrices $S_{1}, S_{2}, T_{1}, T_{2}, T_{3}, M_{1}$, and $M_{2}$ are to be designed. Also, from (13) it is known that

$$
-\phi_{s}^{T}(u(t)) \phi_{s}(u(t))-\phi_{s}^{T}(u(t)) F(e(t)+x(t)) \geqslant 0 .
$$

Then we have

$$
\begin{aligned}
\dot{V}(t)+ & 2 \alpha V(t) \\
\leqslant & 2 \dot{x}^{T}(t) P_{1} x(t)+2 \alpha x^{T}(t) P_{1} x(t) \\
& -e^{-2 \alpha \tau(t)} \tau(t) \eta_{x}^{T}(t) Q_{2} \eta_{x}(t) \\
& +\left(h-t+t_{k}\right) \dot{x}^{T}(t) Q_{2} \dot{x}(t) \\
& -\left(x\left(t_{k}\right)-x(t)\right)^{T} Q_{3}\left(x\left(t_{k}\right)-x(t)\right) \\
& -\left(h-t+t_{k}\right) \dot{x}^{T}(t) Q_{3}\left(x\left(t_{k}\right)-x(t)\right) \\
& -\left(h-t+t_{k}\right)\left(x\left(t_{k}\right)-x(t)\right)^{T} Q_{3} \dot{x}(t) \\
& +2 \alpha\left(h-t+t_{k}\right)\left(x\left(t_{k}\right)-x(t)\right)^{T} \\
& \times Q_{3}\left(x\left(t_{k}\right)-x(t)\right) \\
& +2 \dot{e}^{T}(t) P_{1} e(t)+2 \alpha e^{T}(t) P_{1} e(t) \\
& +2\left[e^{T}(t) S_{1}^{T}+\dot{e}^{T}(t) S_{2}^{T}\right] \\
& \times\left[\dot{e}(t)-(A-L C) e(t)-L C\left(x\left(t_{k}\right)-x(t)\right)\right] \\
& \times 2\left[x^{T}(t) T_{1}^{T}+\dot{x}^{T}(t) T_{2}^{T}+x^{T}\left(t_{k}\right) T_{3}^{T}\right] \\
& \times\left[-x(t)+x\left(t_{k}\right)+\left(t-t_{k}\right) \eta_{x}(t)\right]
\end{aligned}
$$

$$
\begin{aligned}
& \times 2\left[x^{T}(t) M_{1}^{T}+\dot{x}^{T}(t) M_{2}^{T}\right] \\
& \times\left[\dot{x}(t)-\left(A+B F_{1} K\right) x(t)\right. \\
& \left.\quad-B F_{1} K e(t)-B \phi_{s}(u(t))\right] \\
& -\phi_{s}^{T}(u(t)) \phi_{s}(u(t))-\phi_{s}^{T}(u(t)) F(e(t)+x(t)) .
\end{aligned}
$$

Define the following new augmented variables:

$$
\begin{aligned}
& \xi(t)=\left[\begin{array}{lllllll}
x^{T}(t) & \dot{x}^{T}(t) & x^{T}\left(t_{k}\right) & \eta_{x}^{T}(t) & e^{T}(t) & \dot{e}^{T}(t) & \phi_{s}^{T}(u(t))
\end{array}\right]^{T}, \\
& \psi(t)=\left[\begin{array}{llllll}
x^{T}(t) & \dot{x}^{T}(t) & x^{T}\left(t_{k}\right) & e^{T}(t) & \dot{e}^{T}(t) & \phi_{s}^{T}(u(t))
\end{array}\right]^{T} .
\end{aligned}
$$

It is calculated that

$$
\dot{V}(t)+2 \alpha V(t) \leqslant \xi^{T}(t) \Phi(t) \xi(t)
$$

with

$\Phi(t)$

$$
\begin{aligned}
& =\left[\begin{array}{ccccccc}
\Phi_{11}(t) & \Phi_{12}(t) & \Phi_{13}(t) & \Phi_{14}(t) & \Phi_{15}(t) & 0 & \Phi_{17}(t) \\
\star & \Phi_{22}(t) & \Phi_{23}(t) & \Phi_{24}(t) & \Phi_{25}(t) & 0 & \Phi_{27}(t) \\
\star & \star & \Phi_{33}(t) & \Phi_{34}(t) & \Phi_{35}(t) & 0 & 0 \\
\star & \star & \star & \Phi_{44}(t) & 0 & 0 & 0 \\
\star & \star & \star & \star & \Phi_{55}(t) & \Phi_{56}(t) & \Phi_{57}(t) \\
\star & \star & \star & \star & \star & \Phi_{66}(t) & \Phi_{67}(t) \\
\star & \star & \star & \star & \star & \star & \Phi_{77}(t)
\end{array}\right], \\
& \Phi_{11}=2 \alpha Q_{1}+2 \alpha\left(h-t+t_{k}\right) Q_{3}-T_{1}-T_{1}^{T} \\
& -M_{1}^{T}\left(A+B F_{1} K\right)-\left(A+B F_{1} K\right)^{T} M_{1}, \\
& \Phi_{12}=Q_{1}+\left(h-t+t_{k}\right) Q_{3}+M_{1}^{T}, \\
& \Phi_{13}=Q_{3}-2 \alpha\left(h-t+t_{k}\right) Q_{3}+T_{1}^{T} \text {, } \\
& \Phi_{14}=T_{1}^{T}\left(h-t+t_{k}\right) \eta_{x}(t), \\
& \Phi_{15}=S_{1}-M_{1}^{T} B F_{1} K \text {, } \\
& \Phi_{17}=K^{T} F^{T}-M_{1}^{T} B_{1} \text {, } \\
& \Phi_{22}=M_{2}+M_{2}^{T}+\left(h-t+t_{k}\right) Q_{2} \text {, } \\
& \Phi_{23}=-\left(h-t+t_{k}\right) Q_{3}+T_{2}^{T}, \\
& \Phi_{24}=\left(t-t_{k}\right) T_{2}^{T}, \\
& \Phi_{25}=-M_{2}^{T} B F_{1} K \text {, } \\
& \Phi_{27}=-0.5 M_{2}^{T} B \text {, }
\end{aligned}
$$




$$
\begin{aligned}
& \Phi_{33}=-Q_{3}+2 \alpha\left(h-t-t_{k}\right) Q_{3}+T_{3}+T_{3}^{T}, \\
& \Phi_{34}=\left(t+t_{k}\right) T_{3}^{T}, \\
& \Phi_{35}=-C^{T} L^{T} S_{1}, \\
& \widehat{\Phi}_{44}=-e^{-2 \alpha\left(t-t_{k}\right)}\left(t-t_{k}\right) Q_{2}, \\
& \widehat{\Phi}_{55}=2 \alpha P_{1}-S_{1}^{T}(A-L C)-(A-L C)^{T} S_{1}^{T}, \\
& \widehat{\Phi}_{56}=P_{1}+S_{1}^{T}, \\
& \widehat{\Phi}_{57}=0.5 K^{T} F^{T}, \\
& \widehat{\Phi}_{66}=S_{2}+S_{2}^{T} .
\end{aligned}
$$

We now consider the matrices $\Pi$ and $\widehat{\Pi}$ defined in (19) and (20). In fact, it can be verified that $\Pi$ is obtained from $\left.\Phi(t)\right|_{t-t_{k}=0}$ with deleting the 4 th row and the 4 th column, and $\widehat{\Pi}=\left.\Phi(t)\right|_{\mathcal{\tau}(t)=h}$. Similar to the proof of Theorem 1 of [34], it can be proved that the system (17) is exponentially stable.

Next, we deal with the input constraint along each axis. We define

$$
\begin{aligned}
& R_{x}=\left[\begin{array}{l}
1 \\
0 \\
0
\end{array}\right]\left[\begin{array}{lll}
1 & 0 & 0
\end{array}\right]=\left[\begin{array}{lll}
1 & 0 & 0 \\
0 & 0 & 0 \\
0 & 0 & 0
\end{array}\right], \\
& R_{y}=\left[\begin{array}{l}
0 \\
1 \\
0
\end{array}\right]\left[\begin{array}{lll}
0 & 1 & 0
\end{array}\right]=\left[\begin{array}{lll}
0 & 0 & 0 \\
0 & 1 & 0 \\
0 & 0 & 0
\end{array}\right], \\
& R_{z}=\left[\begin{array}{l}
0 \\
0 \\
1
\end{array}\right]\left[\begin{array}{lll}
0 & 0 & 1
\end{array}\right]=\left[\begin{array}{lll}
0 & 0 & 0 \\
0 & 0 & 0 \\
0 & 0 & 1
\end{array}\right] .
\end{aligned}
$$

Then, the thrust along each axis can be described as

$$
\left|u_{i}(t)\right|=\left\|R_{i} u(t)\right\| \leqslant u_{i, \max }, \quad i=x, y, z .
$$

Therefore, the thrust constraint (10) can be rewritten as

$$
\left\|R_{i} u\left(t_{k}\right)\right\| \leqslant u_{i, \max }, \quad i=x, y, z,
$$

which is equivalent to

$$
\left(R_{i} u\left(t_{k}\right)\right)^{T} R_{i} u\left(t_{k}\right) \leqslant u_{i, \text { max }}^{2}
$$

Furthermore, formula (45) is equal to

$$
\widehat{x}^{T}(t) K R_{i}^{T} R_{i} K \widehat{x}(t) \leqslant u_{i, \max }^{2}
$$

which is further equivalent to

$$
\begin{aligned}
& {\left[\begin{array}{ll}
x^{T}(t) & e^{T}(t)
\end{array}\right]\left[\begin{array}{cc}
u_{i, \max }^{-2} K^{T} R_{i}^{T} R_{i} K & u_{i, \max }^{-2} K^{T} R_{i}^{T} R_{i} K \\
\star & u_{i, \max }^{-2} K^{T} R_{i}^{T} R_{i} K
\end{array}\right]} \\
& \times\left[\begin{array}{l}
x(t) \\
e(t)
\end{array}\right] \leqslant 1 .
\end{aligned}
$$

On the other hand, based on the previous analysis, it is known that $\dot{V}(t)<0$ is guaranteed if the matrix conditions (19) and (20) hold. Subsequently, $V(t)<V(0)$ holds for any $t>0$. It is therefore reasonable to assume that there exists a scalar $\rho>0$ such that

$$
V(0) \leqslant \rho .
$$

Notice that $V(t)=V_{1}(t)+V_{2}(t)+V_{3}(t)+V_{4}(t) \leqslant V(0) \leqslant \rho$, and $V_{2}(t) \geqslant 0, V_{3}(t) \geqslant 0$. Therefore, it is true that

$$
V(t) \leqslant V_{1}(t)+V_{4}(t)<V(0) \leqslant \rho,
$$

which implies that

$$
V_{1}(t)+V_{4}(t)<\rho .
$$

In fact, (50) can be rewritten as

$$
\left[x^{T}(t) e^{T}(t)\right]\left[\begin{array}{cc}
\rho^{-1} Q_{1} & 0 \\
0 & \rho^{-1} P_{1}
\end{array}\right]\left[\begin{array}{l}
x(t) \\
e(t)
\end{array}\right] \leqslant 1 .
$$

It is noted that, if condition (21) holds, then

$$
\begin{aligned}
& {\left[\begin{array}{ll}
x^{T}(t) & e^{T}(t)
\end{array}\right]\left[\begin{array}{ccc}
u_{i, \max }^{-2} K^{T} R_{i}^{T} R_{i} K & u_{i, \max }^{-2} K^{T} R_{i}^{T} R_{i} K \\
\star & & u_{i, \max }^{-2} K^{T} R_{i}^{T} R_{i} K
\end{array}\right]\left[\begin{array}{c}
x(t) \\
e(t)
\end{array}\right]} \\
& <\left[\begin{array}{ll}
x^{T}(t) & e^{T}(t)
\end{array}\right]\left[\begin{array}{cc}
\rho^{-1} Q_{1} & 0 \\
\star & \rho^{-1} P_{1}
\end{array}\right]\left[\begin{array}{c}
x(t) \\
e(t)
\end{array}\right] \leqslant 1 .
\end{aligned}
$$

It can be implied from (52) that

$$
\begin{aligned}
& \widehat{x}^{T}(t) K R_{i}^{T} R_{i} K \hat{x}(t) \\
& \quad<u_{i, \max }^{2}\left[x^{T}(t) e^{T}(t)\right]\left[\begin{array}{cc}
\rho^{-1} Q_{1} & 0 \\
\star & \rho^{-1} P_{1}
\end{array}\right]\left[\begin{array}{c}
x(t) \\
e(t)
\end{array}\right] \\
& \quad \leqslant u_{i, \max }^{2} .
\end{aligned}
$$

As a result, the thrust limitation (10) can be guaranteed. This completes the proof.

In Theorem 2, the sufficient condition under which the observer-based controller exists has been established. However, the performance index function (18) has not been considered there. In the following discussion, we will investigate the design problem of guarantee cost controller. We now, in the performance index function (18), notice that $u(t)=$ $K(e(t)+x(t))$; thus

$$
\begin{aligned}
J(t)=\int_{0}^{\infty}[ & x^{T}(t) U x(t) \\
& \left.+\left(e^{T}(t)+x^{T}(t)\right)^{T} W(e(t)+x(t))\right] d t .
\end{aligned}
$$

We present the following theorem, which presents a sufficient condition for the existence of the guaranteed cost controller for the closed-loop system (17).

Theorem 3. Consider the closed-loop system (17) with $K$ and $L$ designed previously, given a decay rate $\alpha>0$; if there exist positive definite matrices $Q_{1}, Q_{2}, Q_{3}$, and $P_{1} \in \mathbb{R}^{9 \times 9}$ and matrices $S_{1}, S_{2}, T_{1}, T_{2}$, and $T_{3} \in \mathbb{R}^{9 \times 9}$, such that the matrix conditions (19), (20), and (21) and the following conditions hold 


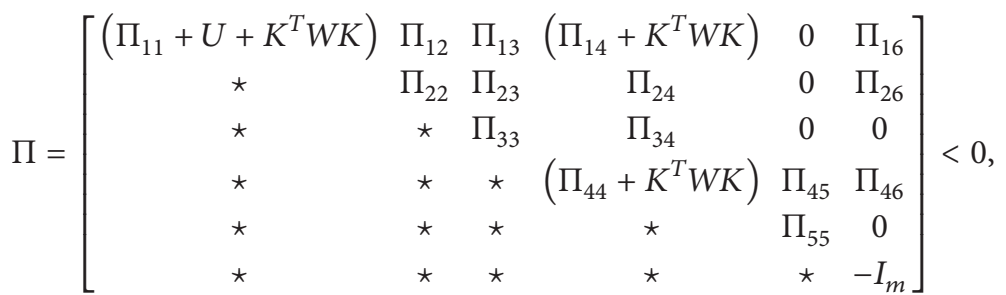

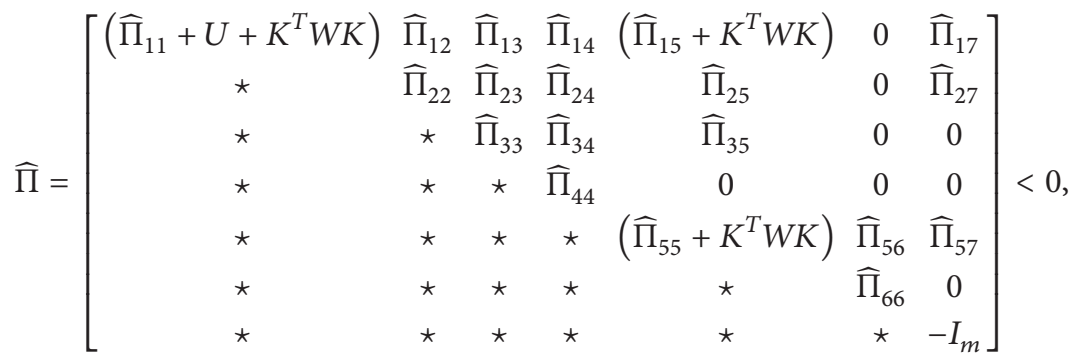

where all the symbols are as previously defined, then system (17) is exponentially stable with the decay rate $\alpha>0$, and the performance index function (18) satisfies

$$
J(t) \leqslant V(0) .
$$

Proof. Consider the term

$$
\begin{aligned}
\Sigma(t)= & \dot{V}(t)+x^{T}(t) U x(t) \\
& +\left(e^{T}(t)+x^{T}(t)\right)^{T} W(e(t)+x(t)),
\end{aligned}
$$

where $V(t)$ is defined as in (4). It is known from Theorem 2 that, if conditions (19), (20), and (21) hold, then $\dot{V}(t)$ is guaranteed for $\forall t>0$. On the other hand, if (55) holds, it is easy to prove that $\Sigma(t)<0$ for $\forall t>0$. Integrating both sides of (57) yields

$$
\begin{aligned}
\int_{0}^{\infty}\left[\dot{V}(t)+x^{T}(t) U x(t)\right. \\
\left.\quad+\left(e^{T}(t)+x^{T}(t)\right)^{T} W(e(t)+x(t))\right] d t<0,
\end{aligned}
$$

or equivalently

$$
\int_{0}^{\infty}[\dot{V}(t) d t+J(t)] d t<0
$$

Therefore, one can obtain

$$
V(\infty)-V(0)+J(t)<0 .
$$

Since $\dot{V}(t)<0, V(\infty)=0$ holds. Hence, it can be concluded that $J(t)<V(0)$ for $\forall t>0$. This completes the proof.

\section{Simulation}

In this section, a simulation example will be shown to illustrate the usefulness and advantage of the proposed sampleddata control approach. We suppose that the mass of the chaser is $200 \mathrm{~kg}$, and the target is moving along a geosynchronous orbit with radius $r=42241 \mathrm{~km}$ with an orbital period of $24 \mathrm{~h}$. The angle velocity $n=1.117 \times 10^{3} \mathrm{rad} / \mathrm{s}$. Suppose that $u_{i, \max }=50$, for $i=x, y, z$ (the maximum thrust along each axis). The angle velocity $n=1.117 \times 10^{-3} \mathrm{rad} / \mathrm{s}$. In the coordinate based on the target frame, assume that the initial state is $(8,-5,5,0,0,0,0,-10,0)$, and the desired holding position is $(0,-2,0)$. The matrices involved with control thrust nonlinearity are chosen as $F_{1}=\operatorname{diag}\{0.6,0.7,0.6\}$, $F_{2}=\operatorname{diag}\{0.8,0.8,0.8\}$; the matrices $U$ and $W$ related to performance index (18) are chosen as $U=\operatorname{diag}\{1,1,1,1,1,1\}$, $W=\operatorname{diag}\{1,1,1\}$; the upper bound of the sampling period is $h=1 \mathrm{~s}$; the decay rate is chosen as $\alpha=0.02$. Design the state feedback gain $K$ and observer gain $L$ as

$$
\begin{gathered}
K=\left[\begin{array}{cccccc}
-5.1092 & 0.0551 & 0.1223 & -51.1446 & -0.0213 & 1.2489 \\
-0.0532 & -4.7752 & 0.0315 & -0.0182 & -45.7196 & 0.3244 \\
-0.4859 & -0.1290 & -5.9323 & -0.6554 & -0.1980 & -52.3649
\end{array}\right], \\
L=\left[\begin{array}{cccc}
0.5332 & 0.0002 & 0 \\
-0.0001 & 0.5 & 0 \\
0 & 0 & 0.521 \\
1.4345 & 0.0001 & 0 \\
0.0016 & 1 & 0 \\
0 & 0 & 0.7018
\end{array}\right]
\end{gathered}
$$




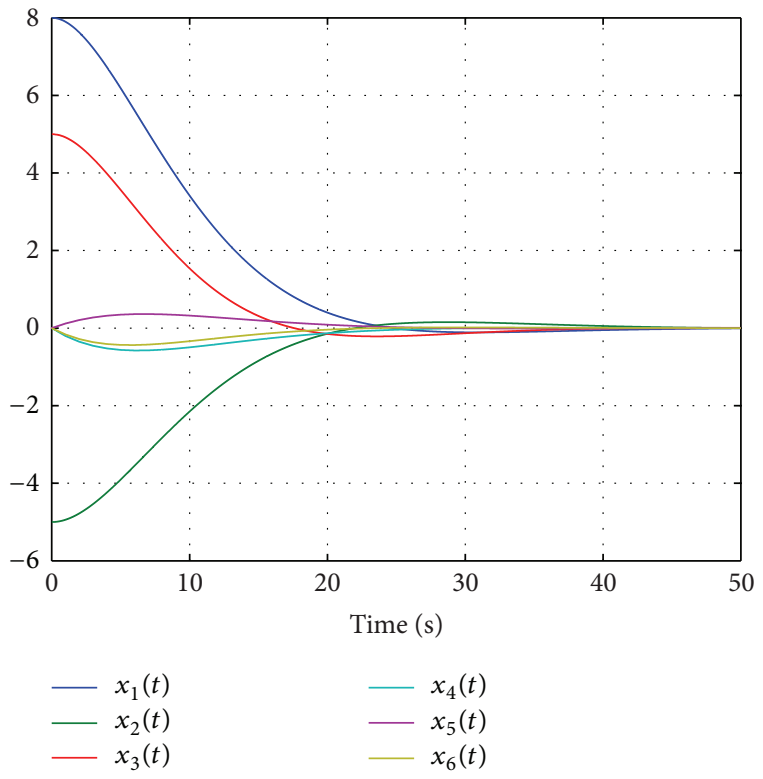

FIGURE 1: Trajectories of $x(t)$.

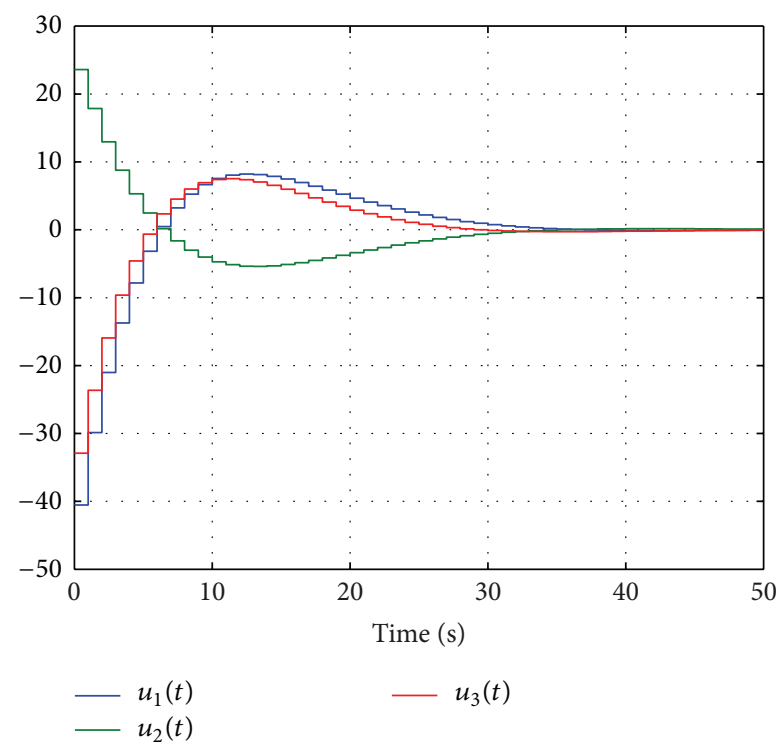

Figure 2: Trajectories of $u(t)$.

such that both $(A+B K)$ and $(A-L C)$ are Hurwitz. Suppose that $t_{1}=0$, which means that the first sampling time instant is the initial time. The designed parameter $\rho$ is chosen as $\rho=30$. Solving the matrices conditions (19), (20), and (21) in Theorem 2 and (55) in Theorem 3, it is found that there exists feasible solution. Then, it can be derived that $V(0)=$ $V_{1}(0)=x(0)^{T} P_{1} x(0)=15.7062$. Therefore, the condition $(48)(V(0) \leqslant \rho)$ holds in this case, and the obtained solution is correct and feasible. The simulation results are provided in Figures 1, 2, 3, and 4, which show that the trajectory of state vector is asymptotically stable.

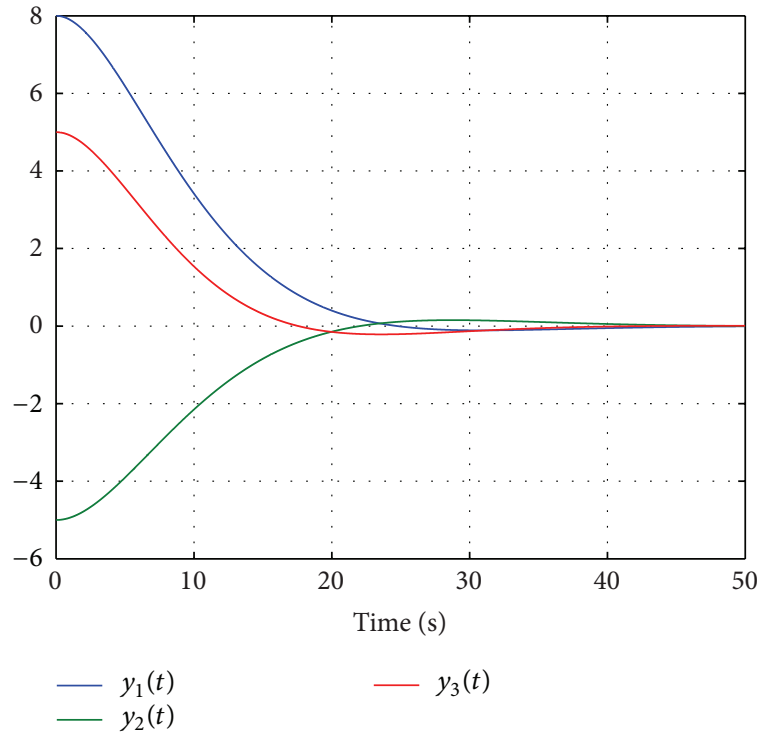

FIGURE 3: Trajectories of $y(t)$.

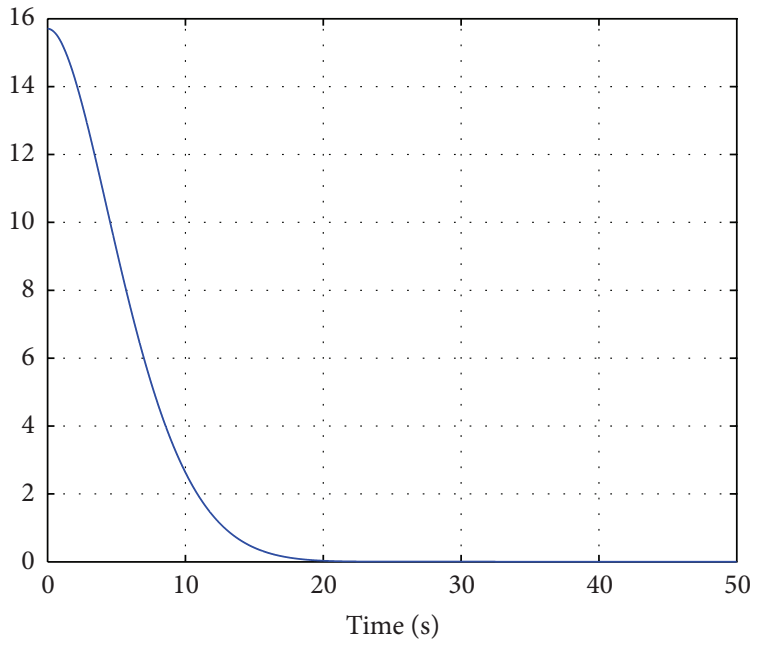

$-V(t)$

Figure 4: Trajectories of $V(t)$.

\section{Conclusion}

In this paper, we have investigated the observer-based sampled-data control problem for spacecraft rendezvous with sensor nonlinearity. The classical Clohessy-Wiltshire equation is used to describe the mathematical model of spacecraft rendezvous. A discontinuous Lyapunov functional approach is presented to pursue a less conservative condition for the stability analysis and controller design. Future work will focus on more complicated cases; for instance, the effects of communication delay, packet losses, and signal sampling are taken into account simultaneously. 


\section{Acknowledgments}

This work was partially supported by the National Natural Science Foundation of China 61104101, the Fundamental Research Funds for the Central Universities, the China Postdoctoral Science Foundation funded project (2011M500058), the Special Chinese National Postdoctoral Science Foundation under Grant 2012T50356, and the Heilongjiang Postdoctoral Fund (LBH-Z11144).

\section{References}

[1] J. Qiu, G. Feng, and H. Gao, "Asynchronous output-feedback control of networked nonlinear systems with multiple packet dropouts: T-S fuzzy affine model-based approach," IEEE Transactions on Fuzzy Systems, vol. 19, no. 6, pp. 1014-1030, 2011.

[2] J. Qiu, G. Feng, and H. Gao, "Nonsynchronized-State estimation of multichannel networked nonlinear systems with multiple packet dropouts via TS Fuzzy-Affine dynamic models," IEEE Transactions on Fuzzy Systems, vol. 19, no. 1, pp. 75-90, 2011.

[3] J. Qiu, G. Feng, and H. Gao, "Fuzzy-model-based piecewise $H_{\infty}$ static-output-feedback controller design for networked nonlinear systems,' IEEE Transactions on Fuzzy Systems, vol. 18, no. 5, pp. 919-934, 2010.

[4] Z. Mao and B. Jiang, "Fault identification and fault-tolerant control for a class of networked control systems," International Journal of Innovative Computing, Information and Control, vol. 3, no. 5, pp. 1121-1130, 2007.

[5] Z.-G. Wu, J. H. Park, H. Su, and J. Chu, "Discontinuous Lyapunov functional approach to synchronization of time-delay neural networks using sampled-data," Nonlinear Dynamics, vol. 69, no. 4, pp. 2021-2030, 2012.

[6] H. Dong, Z. Wang, and H. Gao, " $H_{\infty}$ fuzzy control for systems with repeated scalar nonlinearities and random packet losses," IEEE Transactions on Fuzzy Systems, vol. 17, no. 2, pp. 440-450, 2009.

[7] H. Dong, Z. Wang, D. W. C. Ho, and H. Gao, "Robust $H_{\infty}$ fuzzy output-feedback control with multiple probabilistic delays and multiple missing measurements," IEEE Transactions on Fuzzy Systems, vol. 18, no. 4, pp. 712-725, 2010.

[8] H. Dong, Z. Wang, J. Lam, and H. Gao, "Fuzzy-model-based robust fault detection with stochastic mixed time delays and successive packet dropouts," IEEE Transactions on Systems, Man, and Cybernetics, vol. 42, no. 2, pp. 365-376, 2012.

[9] L.-S. Hu, J. Lam, Y.Y. Cao, and H.-H. Shao, "A linear matrix inequality (LMI) approach to robust $\mathrm{H}_{2}$ sampled-data control for linear uncertain systems," IEEE Transactions on Systems, Man, and Cybernetics, vol. 33, no. 1, pp. 149-155, 2003.

[10] L. Zhang and E.-K. Boukas, "Stability and stabilization of Markovian jump linear systems with partly unknown transition probabilities," Automatica, vol. 45, no. 2, pp. 463-468, 2009.

[11] L. Zhang and E.-K. Boukas, "Mode-dependent $H_{\infty}$ filtering for discrete-time Markovian jump linear systems with partly unknown transition probabilities," Automatica, vol. 45, no. 6 , pp. 1462-1467, 2009.

[12] E. Fridman, "A refined input delay approach to sampled-data control," Automatica, vol. 46, no. 2, pp. 421-427, 2010.

[13] E. Fridman, A. Seuret, and J.-P. Richard, "Robust sampleddata stabilization of linear systems: an input delay approach," Automatica, vol. 40, no. 8, pp. 1441-1446, 2004.
[14] M. Liu, J. You, and X. Ma, " $H_{\infty}$ filtering for sampled-data stochastic systems with limited capacity channel," Signal Processing, vol. 91, no. 8, pp. 1826-1837, 2011.

[15] P. Naghshtabrizi, J. P. Hespanha, and A. R. Teel, "Exponential stability of impulsive systems with application to uncertain sampled-data systems," Systems \& Control Letters, vol. 57, no. 5, pp. 378-385, 2008.

[16] P. Gurfil, "Nonlinear feedback control of low-thrust orbital transfer in a central gravitational field," Acta Astronautica, vol. 60, no. 8-9, pp. 631-648, 2007.

[17] S. Kurnaz, O. Cetin, and O. Kaynak, "Fuzzy logic based approach to design of flight control and navigation tasks for autonomous unmanned aerial vehicles," Journal of Intelligent and Robotic Systems, vol. 54, no. 1-3, pp. 229-244, 2009.

[18] W. Clohessy and R. Wiltshire, "Terminal guidance system for satellite rendezvous," Journal of the Aerospace Sciences, vol. 27, no. 9, pp. 653-658, 1960.

[19] H. Gao, X. Yang, and P. Shi, "Multi-objective robust $H_{\infty}$ control of spacecraft rendezvous," IEEE Transactions on Control Systems Technology, vol. 17, no. 4, pp. 794-802, 2009.

[20] Y.-Z. Luo and G.-J. Tang, "Spacecraft optimal rendezvous controller design using simulated annealing," Aerospace Science and Technology, vol. 9, no. 8, pp. 732-737, 2005.

[21] Y.-Z. Luo, G.-J. Tang, and Y.-J. Lei, "Optimal multi-objective linearized impulsive rendezvous," Journal of Guidance, Control, and Dynamics, vol. 30, no. 2, pp. 383-389, 2007.

[22] X. Yang, X. Cao, and H. Gao, "Sampled-data control for relative position holding of spacecraft rendezvous with thrust nonlinearity," IEEE Transactions on Industrial Electronics, vol. 59, no. 2, pp. 1146-1153, 2012.

[23] S. Yin, S. Ding, and H. Luo, "Real-time implementation of fault tolerant control system with performance optimization," IEEE Transactions on Industrial Electronics, 2013.

[24] S. Yin, S. Ding, A. Haghani, H. Hao, and P. Zhang, "A comparison study of basic data-driven fault diagnosis and process monitoring methods on the benchmark Tennessee Eastman process," Journal of Process Control, vol. 22, no. 9, pp. 1567-1581, 2012.

[25] S. Yin, Data-Driven Design of Fault Diagnosis Systems, VDI, Duesseldorf, Germany, 2012.

[26] S. Yin, S. X. Ding, A. H. A. Sari, and H. Hao, "Data-driven monitoring for stochastic systems and its application on batch process," International Journal of Systems Science, vol. 44, no. 7, pp. 1366-1376, 2013.

[27] L. Wu and W. X. Zheng, "Passivity-based sliding mode control of uncertain singular time-delay systems," Automatica, vol. 45, no. 9, pp. 2120-2127, 2009.

[28] L. Wu and D. W. C. Ho, "Sliding mode control of singular stochastic hybrid systems," Automatica, vol. 46, no. 4, pp. 779783, 2010.

[29] S. Yin, L. Yu, and W. Zhang, "Estimator-based control of networked systems with packet-dropouts," International Journal of Innovative Computing, Information and Control, vol. 6, no. 6, pp. 2737-2748, 2010.

[30] C. Jiang, Q. Zhang, and D. Zou, "Delay-dependent robust filtering for networked control systems with polytopic uncertainties," International Journal of Innovative Computing, Information and Control, vol. 6, no. 11, pp. 4857-4868, 2010.

[31] S. Shi, Z. Yuan, and Q. Zhang, "Fault-tolerant $H_{\infty}$ filter design of a class of switched systems with sensor failures," International Journal of Innovative Computing, Information and Control, vol. 5, no. 11, pp. 3827-3838, 2009. 
[32] H. Wu and M. Bai, "Stochastic stability analysis and synthesis for nonlinear fault tolerant control systems based on the T-S fuzzy model," International Journal of Innovative Computing, Information and Control, vol. 6, no. 9, pp. 3989-4000, 2010.

[33] L. Zhou and G. Lu, "Quantized feedback stabilization for networked control systems with nonlinear perturbation," International Journal of Innovative Computing, Information and Control, vol. 6, no. 6, pp. 2485-2496, 2010.

[34] M. Liu, D. W. C. Ho, and Y. Niu, "Observer-based controller design for linear systems with limited communication capacity via a descriptor augmentation method," IET Control Theory \& Applications, vol. 6, no. 3, pp. 437-447, 2012. 


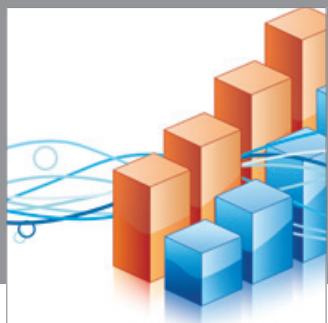

Advances in

Operations Research

mansans

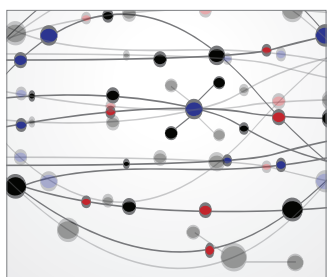

The Scientific World Journal
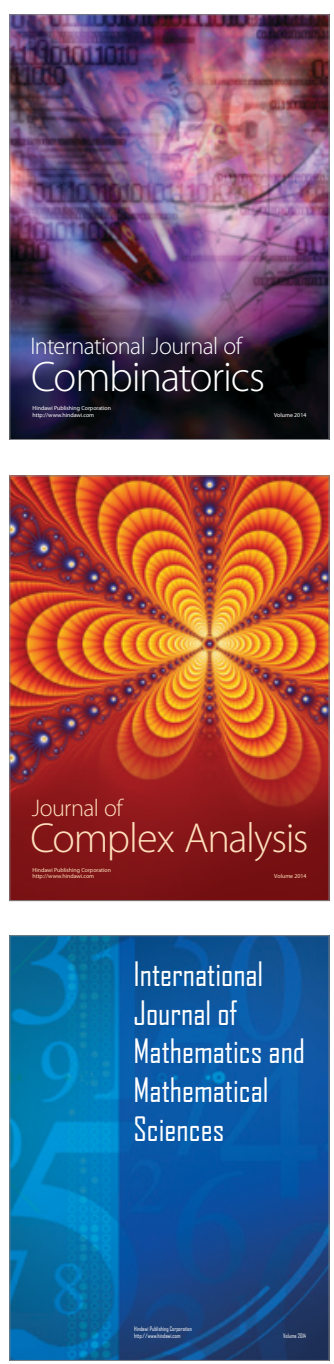
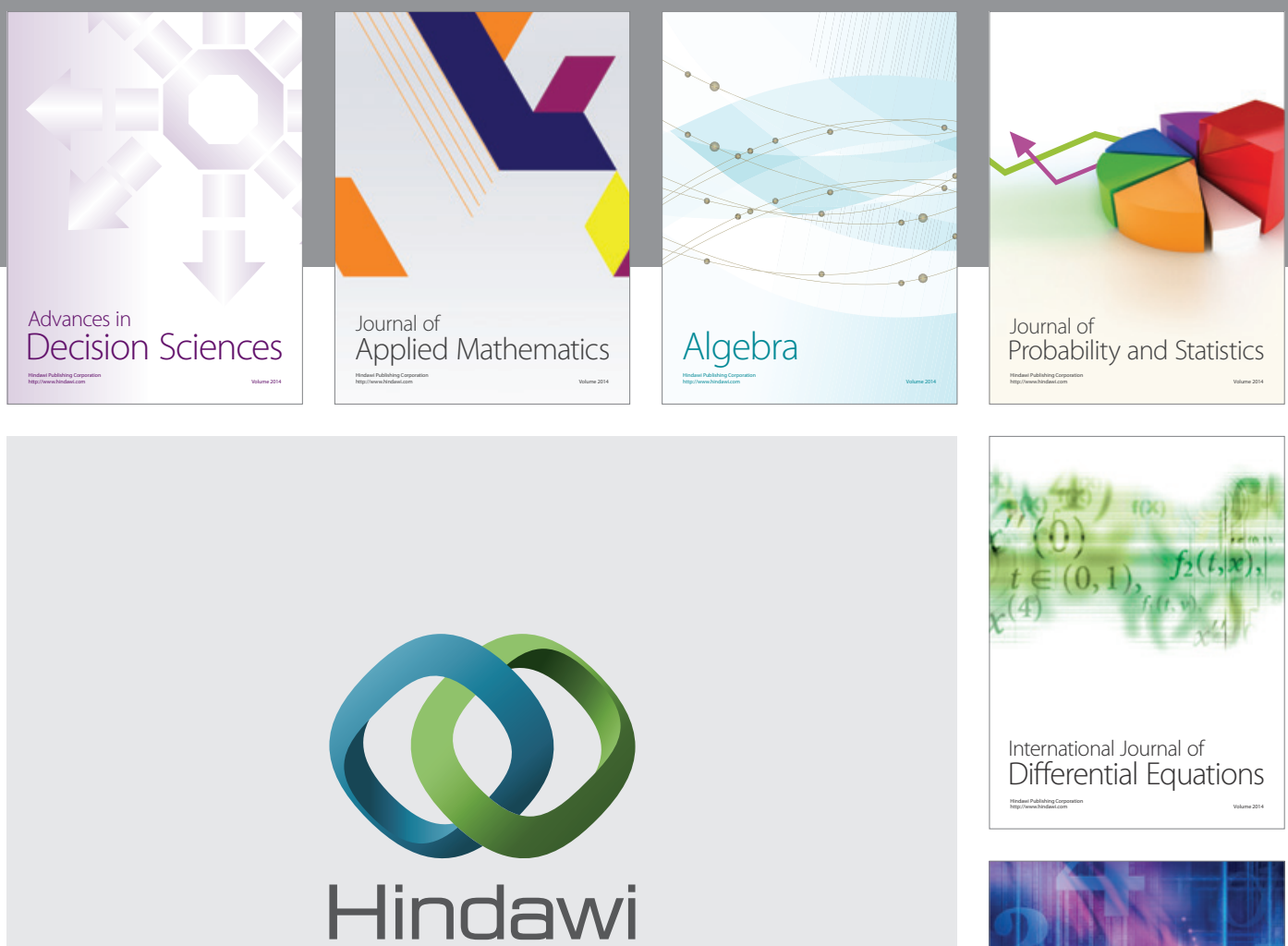

Submit your manuscripts at http://www.hindawi.com
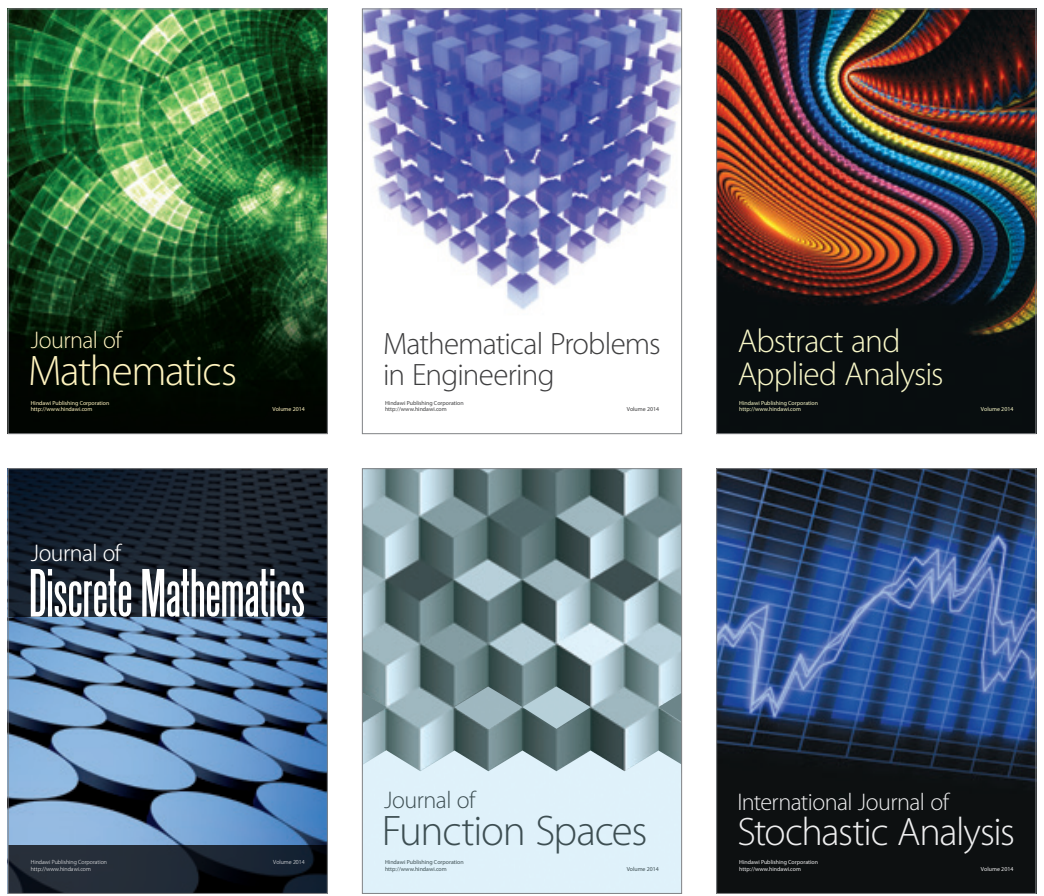

Journal of

Function Spaces

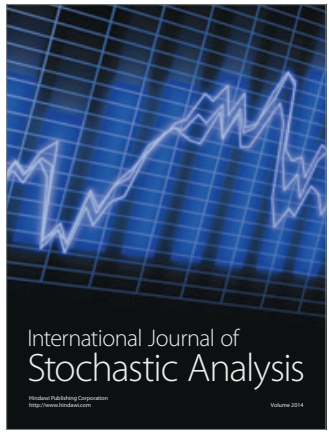

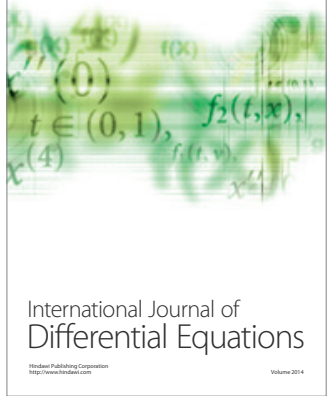
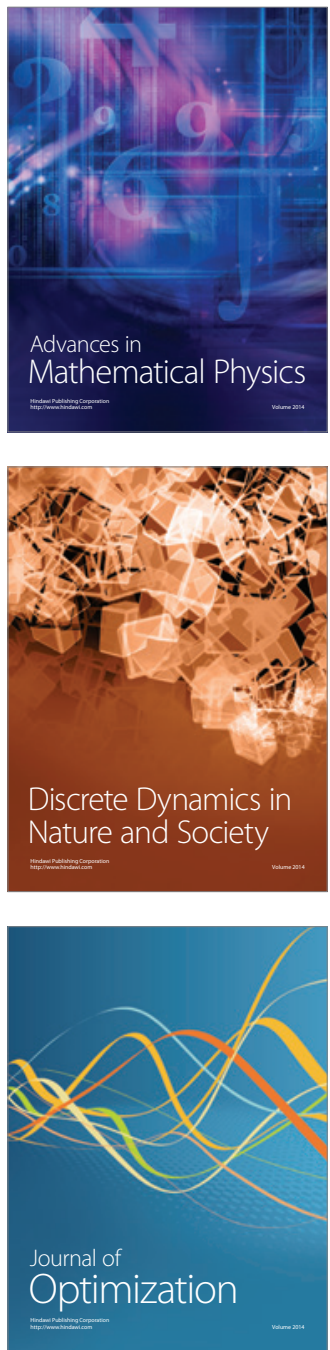